\title{
Pattern and severity of early childhood caries in Southern Italy: a preschool-based cross-sectional study
}

Carmelo GA Nobile, Leonzio Fortunato, Aida Bianco, Claudia Pileggi and Maria Pavia*

\begin{abstract}
Background: This survey was intended to investigate prevalence and severity of early childhood caries (ECC) in a sample of children in Southern Italy and to identify factors that may be related to this condition.

Methods: The study was designed as a cross-sectional survey. The study population (children aged 36-71 months) attending thirteen kindergartens was randomly selected through a two-stage cluster sampling procedure. Parents/guardians of all eligible children were invited to participate filling out a structured self-administered questionnaire, and after having returned the informed consent form an oral examination of the child was performed at school. The questionnaire included information on: socio-demographics about parents/guardians and child, pregnancy and newborn characteristics, oral hygiene habits of child, eating habits particularly on consumption of sweets, access to dental services, and infant feeding practices. The WHO caries diagnostic criteria for deciduous decayed, missing and filled teeth (dmft) and surfaces (dmfs) were used to record ECC and severe-ECC (S-ECC). Univariate and multiple logistic regression analyses were conducted to evaluate statistical associations of social demographics, infant feeding practices, oral hygiene habits, and access to dental services to ECC, S-ECC, dmft and dmfs.
\end{abstract}

Results: 515 children participated in the study. 19\% had experienced ECC, and 2.7\% S-ECC, with a mean dmft and dmfs scores of 0.51 and 0.99 , respectively. Mean dmft was 2.68 in ECC subjects, and 6.86 in S-ECC subjects. Statistical analysis showed that prevalence of ECC significantly increased with age $(\mathrm{OR}=1.95 ; 95 \% \mathrm{Cl}=1.3-2.91)$ and duration of breastfeeding $(\mathrm{OR}=1.26 ; 95 \% \mathrm{Cl}=1.01-1.57)$, whereas it was significantly lower in children of more educated mothers $(\mathrm{OR}=0.64 ; 95 \% \mathrm{Cl}=0.42-0.96)$, and higher in those who had been visited by a dentist in the previous year $(\mathrm{OR}=3.29 ; 95 \% \mathrm{Cl}=1.72-6.33)$.

Conclusions: Results of our study demonstrate that even in Western countries ECC and S-ECC represent a significant burden in preschool children, particularly in those disadvantaged, and that most of the known modifiable associated factors regarding feeding practices and oral hygiene are still very spread in the population.

Keywords: Early childhood caries, Public health, Feeding habits, Children, Prevention

\footnotetext{
* Correspondence: pavia@unicz.it

Department of Health Sciences, Medical School, University of Catanzaro

"Magna Græcia", Via T. Campanella, 115, Catanzaro 88100, Italy
} 


\section{Background}

Although largely preventable and despite the significant improvement of oral health in the past few decades, dental caries remains the most common chronic disease among children and adolescents [1], both in developed and developing countries.

Considering the potential negative impact on overall oral health and on oral health related quality of life of children aged 2-5 years and their parents [2], the early occurrence of dental caries during lifetime, namely early childhood caries (ECC), is of particular concern, since it represents an indicator of preventive missed opportunities, also in developed and developing countries. Moreover, it has been reported that ECC is particularly concentrated in socially disadvantaged children and it has been described as a social, political, behavioral, medical, psychosocial, economical and dental problem [3].

Many definitions of ECC have appeared in the literature, complicating comparisons among different studies and populations. The American Academy of Pediatric Dentistry has then defined ECC as "the presence of 1 or more decayed (noncavitated or cavitated lesions), missing (due to caries), or filled tooth surfaces in any primary tooth in a child 71 months of age or younger" [4]. They also defined severe ECC (S-ECC) with differences according to age: in children younger than 3 years of age, it is defined as any sign of smooth-surface caries, whereas in children from 3 to 5 , it is defined as 1 or more cavitated, missing (due to caries), or filled smooth surfaces in primary maxillary anterior teeth, or a decayed, missing or filled score $(\mathrm{dmft}) \geq 4$ at age 3 , or a dmft $\geq 5$ at age 4 , or a $\mathrm{dmft} \geq 6$ at age 5 [4].

Prevalence of ECC varies among populations, and, within the same population, according to several established or controversial associated factors. It is well established that dietary and biological factors such as frequent consumption of high sugar foods and the presence of bacteria such as Streptococci mutans [5] contribute to ECC development; however 'cariogenic' parental practices are less well understood. Indeed, among parental socio-demographic factors, being of lower social class $[6,7]$, having lower family income [8,9], and living in a single-parent family [10] are contributing factors in the development of dental caries. Education level [11] and living conditions [12] also reflect socio-economic status and may affect knowledge and skills for making health behavior choices and can influence access to health resources. Minority ethnicity [13-15], or immigrant children $[6,16]$, have been found to be at higher risk of caries. Also child feeding practices are important in the early establishment of long term dietary behaviours [17] and short-term development of ECC [18], although controversies still exist. Some studies identified both bottle feeding $[19,20]$ and breastfeeding $[21,22]$ as ECC risk factors, whereas other studies found no effect for either breastfeeding [23-25] or bottle feeding [26] or differences between the two feeding practices [27]. It is also possible that the length of breast and bottle feeding contributes to caries development. Delayed weaning (e.g., between 12 and 36 months) has been found to be associated with caries in various studies [12,28]. However, other studies reported no effect for age at which child was weaned from breast $[23,29,30]$ or bottle [24]. Children who received nocturnal bottle feeding had higher risk for ECC $[19,20,27]$. However, nocturnal breastfeeding, [31] its frequency, particularly if more than twice throughout the night [29,31] and duration > 15 minutes [31] appear to be risk factors. However other studies did not find an association between nocturnal feeding $[20,32,33]$ and caries. Delayed introduction to toothbrushing (e.g., after 12 months of age) is somewhat controversial. Frequency of brushing, $[12,14,33]$ bedtime brushing [33] and time spent brushing [14] were found to be associated with ECC in some studies, however other studies failed to find associations between frequency of toothbrushing and caries [24,26,30,32].

Considering the enormous opportunities of prevention of ECC, effort in the investigation of pattern and severity of ECC, pathways of maladaptive behaviours and poor health outcomes in children are important requisites that can be used to inform the development of evidence-based prevention interventions that target those most at risk.

Although many oral surveys have been conducted to explore the oral health of subgroups of population [34-37], few attention has been posed to ECC and S-ECC.

The primary purpose of this study was to investigate prevalence and severity of ECC; a secondary goal was to identify the extent to which well established or more controversial factors are associated to ECC.

\section{Methods \\ Study design}

The study was designed as a cross-sectional survey. The study population was selected through a two-stage cluster sampling procedure. Thirteen out of 41 kindergartens in the city of Lamezia Terme (CZ) in Southern Italy were randomly selected, and all children aged 3671 months attending these schools were considered eligible. No exclusion criteria of children and parents were established. Parents/guardians of all eligible children were informed through a letter about the purposes and the main issues of the study and invited to participate. The participation consisted, after having returned an informed consent, in the compilation of a structured selfadministered questionnaire by the parent/guardian and on an oral examination of the child performed at school.

The sample size was calculated before study initiation, assuming that ECC prevalence was about 20\% 
in accordance with the literature, a margin of error of $5 \%$, and a $95 \%$ confidence level. Consequently, a sample of 246 children was sought. We decided to be conservative and to inflate our sample size to 640 children by anticipating a response rate of $40 \%$.

\section{Survey instruments}

The questionnaire completed by parents/guardians included information on: socio-demographics about parents/guardians (sex, age, education level) and child (sex, age, birth order, number of brothers/sisters), pregnancy and newborn characteristics (age of mother at delivery, gestational age, birth weight), oral hygiene habits of child (age at first and frequency of toothbrushing, supervision of toothbrushing, use of toothpaste, use of fluoride supplements), eating habits particularly on consumption of sweets, access to dental services (dental problems and visits in the previous year, reasons for dental visits and treatments received), and infant feeding practices (occurrence and duration of breastfeeding, bottle feeding, sleep with a sweetened bottle and/or pacifier, non-nutritive sucking habits with fingers and pacifiers, and age at start using cup).

\section{Oral examinations}

The dental examinations were conducted at school by one trained and calibrated dentist (LF). Duplicate clinical examinations were performed to test intra-examiner reliability until substantial correlation measured by Cohen's Kappa $(k \geq 0.6)$ and a $>90 \%$ Kappa agreement were shown.

Children were seated on a chair facing a window and the examiner used portable equipment consisting of plane mouth mirror, explorer, and a periodontal ball-pointed probe. No radiographs were taken. The WHO caries diagnostic criteria for $\mathrm{dmft}$ and $\mathrm{dmf}$ surfaces (dmfs) [38] were used to record ECC and S-ECC. The prevalence of natural teeth was recorded clinically according to the WHO criteria.

The Ethics Committee of the "Mater Domini" Hospital of Catanzaro (Italy) approved the protocol of the study (Prot. EC no.73/2006).

\section{Statistical analysis}

Univariate analysis was conducted using appropriate tests $\left(\chi^{2}\right.$ and $\chi^{2}$ for trend, Fisher exact test, t-test, Anova) to evaluate associations of social demographics, infant feeding practices, oral hygiene habits, and access to dental services to ECC, S-ECC, dmft and dmfs. Variables that showed significant associations and potential confounders were included in multiple logistic regression models, that were constructed to assess the independent effect of these covariates on prevalence of ECC (Model 1) and S-ECC (Model 2). The variables entered in the models were the following: child's age (months) (ordinal: $0=36-47 ; 1=48-59 ; 2=60-71$ ), mother's age (years) (ordinal: $0=\leq 30 ; 1=31-35 ; 2=36-40 ; 3=\geq 41)$, mother's education level (ordinal: $0=$ none/elementary school; $1=$ junior high school; $2=$ high school; $3=$ university), maternal age at delivery (years) (ordinal: $0=\leq 25 ; 1=26-30$; $2=31-35 ; 3=\geq 36$ ), history and duration of breastfeeding (months) (ordinal: $0=$ no; $1=\leq 4 ; 2=5-10 ; 3=11-19$; $4=\geq 20)$, sleep with sweetened bottle or pacifier $(0=$ no; $1=$ yes), start using cup (months) (ordinal: $0=\leq 12 ; 1=$ $13-18 ; 2=\geq 19$ ), start toothbrushing (months) (ordinal: $0=\leq 12 ; 1=13-24 ; 2=\geq 25)$, dental visit in the previous year $(0=$ no; $1=$ yes $)$.

All analyses were conducted using the Stata version 11 software program [39].

\section{Results}

Of the total 640 eligible children, 515 participated in the study, thus giving a response rate of $80.5 \%$. Nonresponses were due to no-contact (i.e. children were not at the kindergarten when the research team attempted to make first and second contact), not-able (i.e. contacted parents wanted to participate but children were ill at the time of first and second oral examinations), and refusal (i.e. contacted parents refused to answer to the questionnaire for concern of oral examinations or privacy reasons). Children were evenly distributed between males and females, 161 were 3, 196 were 4, and 158 were five years old. Overall, 98 (19\%) had experienced ECC, and $14(2.7 \%)$ S-ECC, with a mean $\mathrm{dmft}$ and $\mathrm{dmfs}$ of 0.51 $(\mathrm{SD} \pm 1.4)$ and $0.99(\mathrm{SD} \pm 3.04)$, respectively. Among those who had ECC, $97 \%$ had untreated decayed teeth. Mean $\mathrm{dmft}$ was $2.68(\mathrm{SD} \pm 2.11)$ in ECC subjects, and 6.86 $(\mathrm{SD} \pm 1.56)$ in $\mathrm{S}$-ECC subjects. ECC pattern and severity according to social demographics, pregnancy and newborn characteristics, infant feeding practices, oral hygiene and eating habits, and access to dental services are reported in Table 1.

As regards to social demographics, age of child, age of the mother at the time of the survey and at time of delivery, and education level of both parents were all significantly associated to ECC pattern. Indeed, at univariate analysis, ECC prevalence significantly increased with age of the child, ranging from $8.7 \%$ in 3 years old children to $29.8 \%$ in 5 years old children $\left(\chi^{2}\right.$ for trend $=22.89$; $\mathrm{p}<$ 0.001 ), whereas it significantly decreased with increasing age of the mother at the time of the survey, ranging from $30.5 \%$ in mothers $\leq 30$ years to $15.2 \%$ in $\geq 41$ years of age $\left(\chi^{2}\right.$ for trend $\left.=4.94 ; \mathrm{p}=0.026\right)$ and at the time of delivery, ranging from $31.4 \%$ in mothers delivering at 25 or younger to $12.7 \%$ in those older than 35 at delivery $\left(\chi^{2}\right.$ for trend $\left.=9.36 ; \mathrm{p}=0.002\right)$; the same significant decreasing trend of ECC prevalence was found for education level of mothers, ranging from $31.3 \%$ in those with 
Table 1 ECC pattern according to newborn characteristics, feeding practices, oral hygiene habits, and access to dental services

\begin{tabular}{|c|c|c|c|c|c|c|c|c|c|c|}
\hline \multirow[t]{2}{*}{ Variable or practice } & \multirow[b]{2}{*}{$\mathrm{N}$} & \multirow[b]{2}{*}{$\%$} & \multicolumn{2}{|c|}{$\mathrm{ECC}$} & \multicolumn{2}{|c|}{ S-ECC } & \multirow{2}{*}{$\begin{array}{c}\mathrm{dmft} \\
\text { Mean } \pm \text { SD }\end{array}$} & \multirow{2}{*}{$\begin{array}{c}d t \\
\text { Mean } \pm \text { SD }\end{array}$} & \multirow{2}{*}{$\begin{array}{c}\text { dmfs } \\
\text { Mean } \pm \text { SD }\end{array}$} & \multirow{2}{*}{$\begin{array}{c}\text { ds } \\
\text { Mean } \pm \text { SD }\end{array}$} \\
\hline & & & $\mathbf{N}$ & $\%$ & $\mathbf{N}$ & $\%$ & & & & \\
\hline \multicolumn{11}{|c|}{ Social demographics and newborn characteristics } \\
\hline \multicolumn{11}{|l|}{ Age, months } \\
\hline $36-47$ & 161 & 31.2 & 14 & 8.7 & 2 & 1.2 & $0.17 \pm 0.78$ & $0.17 \pm 0.78$ & $0.30 \pm 1.65$ & $0.25 \pm 1.6$ \\
\hline $48-59$ & 196 & 38.1 & 37 & 18.9 & 6 & 3.1 & $0.49 \pm 1.38$ & $0.45 \pm 1.29$ & $0.96 \pm 3.09$ & $0.86 \pm 2.98$ \\
\hline \multirow[t]{2}{*}{$60-71$} & 158 & 30.7 & 47 & 29.8 & 6 & 3.8 & $0.89 \pm 1.78$ & $0.86 \pm 1.77$ & $1.71 \pm 3.81$ & $1.68 \pm 3.81$ \\
\hline & & & \multicolumn{2}{|c|}{$x^{2}$ for trend $=22.89 ; p<0.001$} & \multicolumn{2}{|c|}{ Fisher's exact $p=0.324$} & $F=10.99 p<0.001$ & $F=10.86 p<0.001$ & $F=8.78 p<0.001$ & $F=9.40 p<0.001$ \\
\hline \multicolumn{11}{|l|}{ Gender } \\
\hline Male & 262 & 50.9 & 54 & 20.6 & 8 & 3.1 & $0.58 \pm 1.51$ & $0.54 \pm 1.46$ & $1.12 \pm 3.24$ & $1.03 \pm 3.18$ \\
\hline \multirow[t]{2}{*}{ Female } & 253 & 49.1 & 44 & 17.4 & 6 & 2.4 & $0.44 \pm 1.27$ & $0.43 \pm 1.25$ & $0.85 \pm 2.82$ & $0.81 \pm 2.78$ \\
\hline & & & \multicolumn{2}{|c|}{$x^{2}=0.87 ; p=0.352$} & \multicolumn{2}{|c|}{$x^{2}=0.23 ; p=0.634$} & t-test $=-1.15 ; p=0.251$ & t-test $=-0.923 ; p=0.355$ & t-test $=-1 ; p=0.317$ & t-test $=-0.85 ; p=0.395$ \\
\hline \multicolumn{11}{|l|}{ Father's age, years } \\
\hline$\leq 35$ & 177 & 35.1 & 36 & 20.3 & 5 & 2.8 & $0.6 \pm 1.57$ & $0.6 \pm 1.57$ & $1.24 \pm 3.72$ & $1.2 \pm 3.71$ \\
\hline $36-40$ & 160 & 31.7 & 31 & 19.4 & 7 & 4.4 & $0.52 \pm 1.37$ & $0.44 \pm 1.25$ & $1.04 \pm 2.88$ & $0.86 \pm 2.72$ \\
\hline $41-45$ & 125 & 24.7 & 23 & 18.4 & 2 & 1.6 & $0.46 \pm 1.35$ & $0.46 \pm 1.35$ & $0.78 \pm 2.63$ & $0.78 \pm 2.63$ \\
\hline \multirow[t]{2}{*}{$\geq 46$} & 43 & 8.5 & 8 & 18.6 & 0 & - & $0.37 \pm 0.95$ & $0.37 \pm 0.95$ & $0.56 \pm 1.58$ & $0.56 \pm 1.58$ \\
\hline & & & \multicolumn{2}{|c|}{$x^{2}$ for trend $=0.18 ; p=0.676$} & \multicolumn{2}{|c|}{ Fisher's exact $p=0.446$} & $F=0.45 ; p=0.714$ & $F=0.6 ; p=0.618$ & $F=0.87 ; p=0.458$ & $F=0.81 ; p=0.486$ \\
\hline \multicolumn{11}{|l|}{ Mother's age, years } \\
\hline$\leq 30$ & 105 & 20.7 & 32 & 30.5 & 9 & 8.6 & $1.02 \pm 2.12$ & $0.91 \pm 2.02$ & $1.91 \pm 4.91$ & $1.81 \pm 4.86$ \\
\hline $31-35$ & 166 & 32.7 & 24 & 14.5 & 1 & 0.6 & $0.3 \pm 0.86$ & $0.3 \pm 0.86$ & $0.71 \pm 2.07$ & $0.61 \pm 1.95$ \\
\hline $36-40$ & 171 & 33.6 & 31 & 18.1 & 4 & 2.3 & $0.49 \pm 1.39$ & $0.49 \pm 1.39$ & $0.91 \pm 2.73$ & $0.87 \pm 2.7$ \\
\hline \multirow[t]{2}{*}{$\geq 41$} & 66 & 13 & 10 & 15.2 & 0 & - & $0.32 \pm 0.88$ & $0.32 \pm 0.88$ & $0.5 \pm 1.52$ & $0.5 \pm 1.52$ \\
\hline & & & \multicolumn{2}{|c|}{$x^{2}$ for trend $=4.94 ; p=0.026$} & \multicolumn{2}{|c|}{ Fisher's exact $p=0.001$} & $F=6.43 ; p=0.0003$ & $F=4.87 ; p=0.002$ & $F=4.35 ; p=0.005$ & $F=4.16 ; p=0.006$ \\
\hline \multicolumn{11}{|l|}{ Child order } \\
\hline First & 123 & 30.9 & 19 & 15.5 & 4 & 3.3 & $0.46 \pm 1.45$ & $0.41 \pm 1.36$ & $0.96 \pm 3.48$ & $0.88 \pm 3.44$ \\
\hline Second & 213 & 51 & 47 & 23.2 & 5 & 2.5 & $0.56 \pm 1.4$ & $0.54 \pm 1.38$ & $1.08 \pm 3.05$ & $0.99 \pm 2.96$ \\
\hline Third or more & 72 & 18.1 & 12 & 16.7 & 3 & 4.2 & $0.58 \pm 1.55$ & $0.58 \pm 1.55$ & $1.07 \pm 2.74$ & $1.01 \pm 2.72$ \\
\hline & & & $x^{2}$ for $t$ & $p=0.583$ & Fisher's & $=0.683$ & $F=0.25 ; p=0.78$ & $F=0.47 ; p=0.627$ & $F=0.06 ; p=0.939$ & $F=0.07 ; p=0.935$ \\
\hline Father's education level & & & & & & & & & & \\
\hline None/elementary school & 16 & 3.2 & 6 & 37.5 & 1 & 6.3 & $1.19 \pm 2.2$ & $1.19 \pm 2.2$ & $2.25 \pm 5$ & $2.25 \pm 5$ \\
\hline Junior high school & 183 & 36.6 & 45 & 24.6 & 8 & 4.4 & $0.68 \pm 1.6$ & $0.66 \pm 1.59$ & $1.28 \pm 3.2$ & $1.2 \pm 3.15$ \\
\hline High school & 246 & 49.2 & 32 & 13 & 4 & 1.6 & $0.35 \pm 1.18$ & $0.32 \pm 1.09$ & $0.74 \pm 2.83$ & $0.67 \pm 2.75$ \\
\hline
\end{tabular}


Table 1 ECC pattern according to newborn characteristics, feeding practices, oral hygiene habits, and access to dental services (Continued)

\begin{tabular}{|c|c|c|c|c|c|c|c|c|c|c|}
\hline \multirow[t]{2}{*}{ University } & 55 & 11 & 13 & 23.6 & 0 & - & $0.42 \pm 0.9$ & $0.42 \pm 0.9$ & $0.58 \pm 1.4$ & $0.58 \pm 1.4$ \\
\hline & & & \multicolumn{2}{|c|}{$x^{2}$ for trend $=4.52 ; p=0.03$} & \multicolumn{2}{|c|}{ Fisher's exact $p=0.112$} & $F=3.45 ; p=0.016$ & $F=3.87 ; p=0.009$ & $F=2.50 ; p=0.059$ & $F=2.53 ; p=0.056$ \\
\hline \multicolumn{11}{|l|}{ Mother's education level } \\
\hline None/elementary school & 16 & 3.2 & 5 & 31.3 & 1 & 6.3 & $0.69 \pm 1.74$ & $0.69 \pm 1.74$ & $1.19 \pm 3.71$ & $1.19 \pm 3.71$ \\
\hline Junior high school & 141 & 27.8 & 37 & 26.2 & 8 & 5.7 & $0.83 \pm 1.82$ & $0.82 \pm 1.81$ & $1.58 \pm 3.77$ & $1.5 \pm 3.72$ \\
\hline High school & 268 & 53 & 39 & 14.6 & 4 & 1.5 & $0.36 \pm 1.14$ & $0.33 \pm 1.07$ & $0.69 \pm 2.56$ & $0.61 \pm 2.49$ \\
\hline \multirow[t]{2}{*}{ University } & 81 & 16 & 15 & 18.5 & 0 & - & $0.36 \pm 0.88$ & $0.35 \pm 0.88$ & $0.73 \pm 2.07$ & $0.72 \pm 2.08$ \\
\hline & & & \multicolumn{2}{|c|}{$x^{2}$ for trend $=5.10 ; p=0.024$} & \multicolumn{2}{|c|}{ Fisher's exact $p=0.019$} & $F=4.11 ; p=0.007$ & $F=4.65 ; p=0.003$ & $F=3.08 ; p=0.027$ & $F=3.09 ; p=0.027$ \\
\hline \multicolumn{11}{|c|}{ Maternal age at delivery, years } \\
\hline$\leq 25$ & 105 & 20.8 & 33 & 31.4 & 9 & 8.6 & $1.06 \pm 2.12$ & $0.95 \pm 2.03$ & $2.05 \pm 4.9$ & $1.92 \pm 4.86$ \\
\hline $26-30$ & 179 & 35.4 & 31 & 17.3 & 2 & 1.1 & $0.35 \pm 0.95$ & $0.35 \pm 0.95$ & $0.75 \pm 2.2$ & $0.67 \pm 2.11$ \\
\hline $31-35$ & 158 & 31.3 & 23 & 16.5 & 3 & 1.9 & $0.47 \pm 1.38$ & $0.47 \pm 1.38$ & $0.85 \pm 2.68$ & $0.82 \pm 2.65$ \\
\hline \multirow[t]{2}{*}{$\geq 36$} & 63 & 12.5 & 8 & 12.7 & 0 & - & $0.24 \pm 0.73$ & $0.24 \pm 0.73$ & $0.37 \pm 1.21$ & $0.37 \pm 1.21$ \\
\hline & & & \multicolumn{2}{|c|}{$x^{2}$ for trend $=9.36 ; p=0.002$} & \multicolumn{2}{|c|}{ Fisher's exact $p=0.003$} & $F=7.1 ; p<0.001$ & $F=5.48 ; p=0.001$ & $F=5.64 ; p=0.001$ & $F=5.18 ; p=0.002$ \\
\hline \multicolumn{11}{|l|}{ Infant feeding practices } \\
\hline \multicolumn{11}{|c|}{ History and duration of breastfeeding, months } \\
\hline No & 95 & 19.5 & 15 & 15.8 & 1 & 1.1 & $0.38 \pm 1.08$ & $0.38 \pm 1.08$ & $0.69 \pm 2.1$ & $0.69 \pm 2.1$ \\
\hline$\leq 4$ & 150 & 30.8 & 28 & 18.7 & 6 & 4 & $0.59 \pm 1.68$ & $0.56 \pm 1.67$ & $1.25 \pm 4.05$ & $1.19 \pm 4.03$ \\
\hline $5-10$ & 115 & 23.6 & 14 & 12.2 & 0 & - & $0.22 \pm 0.62$ & $0.22 \pm 0.62$ & $0.37 \pm 1.03$ & $0.31 \pm 0.96$ \\
\hline $11-19$ & 62 & 12.7 & 13 & 20.1 & 0 & - & $0.44 \pm 1.07$ & $0.44 \pm 1.07$ & $0.94 \pm 2.47$ & $0.76 \pm 2.26$ \\
\hline \multirow[t]{2}{*}{$\geq 20$} & 65 & 13.4 & 20 & 30.8 & 6 & 9.2 & $1.06 \pm 2.08$ & $1.03 \pm 2.03$ & $2.06 \pm 4.3$ & $2.02 \pm 4.25$ \\
\hline & & & \multicolumn{2}{|c|}{$x^{2}$ for trend $=4.10 ; p=0.043$} & \multicolumn{2}{|c|}{ Fisher's exact $p=0.002$} & $F=4.24 ; p=0.002$ & $F=3.95 ; p=0.004$ & $F=3.66 ; p=0.006$ & $F=3.75 ; p=0.005$ \\
\hline \multicolumn{11}{|c|}{ Non-nutritive sucking habits (finger or pacifier) } \\
\hline No & 473 & 93.8 & 87 & 18.4 & 14 & 2.9 & $0.51 \pm 1.42$ & $0.48 \pm 1.38$ & $1 \pm 3.1$ & $0.93 \pm 3.05$ \\
\hline \multirow[t]{2}{*}{ Yes } & 37 & 7.2 & 9 & 24.3 & 0 & - & $0.49 \pm 1.07$ & $0.49 \pm 1.07$ & $0.76 \pm 2.3$ & $0.76 \pm 2.3$ \\
\hline & & & \multicolumn{2}{|c|}{$x^{2}=0.79 ; p=0.374$} & \multicolumn{2}{|c|}{ Fisher's exact $p=0.613$} & t-test $=0.09 ; p=0.93$ & $\mathrm{t}$-test $=-0.02 ; \mathrm{p}=0.985$ & t-test $=0.47 ; p=0.641$ & t-test $=0.28 ; p=0.781$ \\
\hline \multicolumn{11}{|l|}{ Bottle feeding } \\
\hline No & 79 & 15.7 & 19 & 24.1 & 6 & 7.6 & $0.89 \pm 2.03$ & $0.89 \pm 2.03$ & $1.84 \pm 4.35$ & $1.84 \pm 4.35$ \\
\hline \multirow[t]{2}{*}{ Yes } & 423 & 84.3 & 77 & 18.2 & 8 & 1.9 & $0.44 \pm 1.25$ & $0.41 \pm 1.2$ & $0.84 \pm 2.74$ & $0.76 \pm 2.67$ \\
\hline & & & \multicolumn{2}{|c|}{$x^{2}=1.47 ; p=0.225$} & \multicolumn{2}{|c|}{$x^{2}=7.99 ; p=0.005$} & $\mathrm{t}$-test $=2.66 ; p=0.008$ & t-test $=2.9 ; p=0.004$ & t-test $=2.66 ; p=0.008$ & $\mathrm{t}$-test $=2.93 ; p=0.004$ \\
\hline \multicolumn{11}{|c|}{ Sleep with sweetened bottle or pacifier } \\
\hline No & 412 & 80.8 & 71 & 17.2 & 12 & 2.9 & $0.47 \pm 1.34$ & $0.44 \pm 1.3$ & $0.9 \pm 2.83$ & $0.82 \pm 2.77$ \\
\hline Yes & 98 & 19.2 & 25 & 25.5 & 2 & 2 & $0.67 \pm 1.59$ & $0.65 \pm 1.59$ & $1.35 \pm 3.81$ & $1.31 \pm 3.8$ \\
\hline
\end{tabular}


Table 1 ECC pattern according to newborn characteristics, feeding practices, oral hygiene habits, and access to dental services (Continued)

\begin{tabular}{|c|c|c|c|c|c|c|c|c|c|c|}
\hline & & & \multicolumn{2}{|c|}{$x^{2}=3.55 ; p=0.06$} & \multicolumn{2}{|c|}{ Fisher's exact $p=1$} & t-test $=-1.32 ; p=0.187$ & t-test $=-1.38 ; p=0.167$ & $\mathrm{t}$-test $=-1.32 ; p=0.188$ & $\mathrm{t}$-test $=-1.44 ; p=0.151$ \\
\hline \multicolumn{11}{|l|}{ Start using cup, months } \\
\hline$\leq 12$ & 284 & 59.9 & 46 & 16.2 & 7 & 2.5 & $0.42 \pm 1.3$ & $0.39 \pm 1.24$ & $0.77 \pm 2.59$ & $0.72 \pm 2.56$ \\
\hline $13-18$ & 58 & 12.2 & 11 & 19 & 1 & 1.7 & $0.52 \pm 1.42$ & $0.52 \pm 1.42$ & $1.34 \pm 3.67$ & $1.16 \pm 3.55$ \\
\hline \multirow[t]{2}{*}{$\geq 19$} & 132 & 27.9 & 33 & 25 & 4 & 3 & $0.64 \pm 1.43$ & $0.61 \pm 1.4$ & $1.1 \pm 2.96$ & $1.08 \pm 2.92$ \\
\hline & & & \multicolumn{2}{|c|}{$x^{2}$ for trend $=4.44 ; p=0.035$} & \multicolumn{2}{|c|}{ Fisher's exact $p=0.912$} & $F=1.14 ; p=0.321$ & $F=1.35 ; p=0.26$ & $F=1.31 ; p=0.27$ & $F=1.08 ; p=0.341$ \\
\hline \multicolumn{11}{|l|}{ Sweets consumption } \\
\hline Never & 20 & 4.1 & 1 & 5.0 & 0 & - & $0.05 \pm 0.22$ & $0.05 \pm 0.22$ & $0.05 \pm 0.22$ & $0.05 \pm 0.22$ \\
\hline Less than once a day & 220 & 44.6 & 39 & 17.7 & 6 & 2.7 & $0.46 \pm 1.31$ & $0.43 \pm 1.26$ & $0.82 \pm 2.56$ & $0.77 \pm 2.52$ \\
\hline \multirow[t]{2}{*}{ At least once a day } & 253 & 51.3 & 51 & 20.2 & 8 & 3.2 & $0.58 \pm 1.52$ & $0.56 \pm 1.5$ & $1.19 \pm 3.54$ & $1.09 \pm 3.47$ \\
\hline & & & \multicolumn{2}{|c|}{ Fisher's exact $p=0.241$} & \multicolumn{2}{|c|}{ Fisher's exact $p=1$} & $F=1.51 ; p=0.222$ & $F=1.57 ; p=0.21$ & $F=1.79 ; p=0.168$ & $F=1.54 ; p=0.215$ \\
\hline \multicolumn{11}{|l|}{ Oral hygiene habits } \\
\hline \multicolumn{11}{|l|}{ Toothbrushing habits } \\
\hline Less than once a day & 144 & 28.5 & 35 & 24.3 & 4 & 2.8 & $0.59 \pm 1.49$ & $0.57 \pm 1.46$ & $1.16 \pm 3.28$ & $1.08 \pm 3.23$ \\
\hline Once a day & 210 & 41.5 & 32 & 15.2 & 6 & 2.9 & $0.44 \pm 1.36$ & $0.43 \pm 1.34$ & $0.85 \pm 2.96$ & $0.78 \pm 2.89$ \\
\hline \multirow[t]{2}{*}{ More than once a day } & 152 & 30 & 31 & 20.4 & 4 & 2.6 & $0.56 \pm 1.4$ & $0.51 \pm 1.33$ & $1.07 \pm 3$ & $1.02 \pm 2.98$ \\
\hline & & & \multicolumn{2}{|c|}{$x^{2}$ for trend $=0.67 ; p=0.415$} & \multicolumn{2}{|c|}{ Fisher's exact $p=1$} & $F=0.55 ; p=0.576$ & $F=0.44 ; p=0.647$ & $F=0.47 ; p=0.623$ & $F=0.49 ; p=0.612$ \\
\hline \multicolumn{11}{|c|}{ Start toothbrushing, months } \\
\hline$\leq 12$ & 76 & 15.8 & 14 & 18.4 & 3 & 4 & $0.61 \pm 1.71$ & $0.61 \pm 1.71$ & $1.28 \pm 3.62$ & $1.2 \pm 3.58$ \\
\hline $13-24$ & 219 & 45.4 & 33 & 15.1 & 2 & 0.9 & $0.37 \pm 1.09$ & $0.34 \pm 1.02$ & $0.73 \pm 2.32$ & $0.64 \pm 2.22$ \\
\hline \multirow[t]{2}{*}{$\geq 25$} & 187 & 38.8 & 42 & 22.5 & 7 & 3.7 & $0.61 \pm 1.48$ & $0.58 \pm 1.44$ & $1.1 \pm 3.26$ & $1.05 \pm 3.23$ \\
\hline & & & \multicolumn{2}{|c|}{$x^{2}$ for trend $=1.57 ; p=0.21$} & \multicolumn{2}{|c|}{ Fisher's exact $p=0.087$} & $F=1.91 ; p=0.15$ & $F=2.11 ; p=0.122$ & $F=1.31 p=0.271$ & $F=1.52 ; p=0.221$ \\
\hline \multicolumn{11}{|l|}{ Toothpaste use } \\
\hline No & 32 & 6.4 & 3 & 9.4 & 2 & 6.3 & $0.47 \pm 1.74$ & $0.47 \pm 1.74$ & $0.93 \pm 3.83$ & $0.93 \pm 3.83$ \\
\hline \multirow[t]{2}{*}{ Yes } & 467 & 93.6 & 91 & 19.5 & 12 & 2.6 & $0.52 \pm 1.39$ & $0.49 \pm 1.35$ & $1 \pm 3.02$ & $0.93 \pm 2.97$ \\
\hline & & & \multicolumn{2}{|c|}{ Fisher's exact $p=0.24$} & \multicolumn{2}{|c|}{ Fisher's exact $p=0.224$} & $\mathrm{t}$-test $=-0.19 ; p=0.848$ & $\mathrm{t}$-test $=-0.09 ; \mathrm{p}=0.925$ & $\mathrm{t}$-test $=-0.12 ; p=0.906$ & t-test $=0.01 ; p=0.991$ \\
\hline \multicolumn{11}{|c|}{ Adult supervision of toothbrushing } \\
\hline No & 26 & 5.3 & 5 & 19.2 & 2 & 7.7 & $0.81 \pm 2$ & $0.81 \pm 2$ & $1.62 \pm 4.61$ & $1.62 \pm 4.61$ \\
\hline \multirow[t]{2}{*}{ Yes } & 466 & 94.7 & 85 & 18.2 & 10 & 2.2 & $0.47 \pm 1.3$ & $0.44 \pm 1.26$ & $0.9 \pm 2.79$ & $0.83 \pm 2.72$ \\
\hline & & & \multicolumn{2}{|c|}{$x^{2}=0.02 ; p=0.899$} & \multicolumn{2}{|c|}{ Fisher's exact $p=0.128$} & $\mathrm{t}$-test $=1.24 ; \mathrm{p}=0.214$ & $\mathrm{t}$-test $=1.38 ; \mathrm{p}=0.169$ & $\mathrm{t}$-test $=1.22 ; p=0.224$ & t-test $=1.37 ; p=0.171$ \\
\hline Use of fluoride supplemer & & & & & & & & & & \\
\hline No & 314 & 64.3 & 58 & 18.5 & 7 & 2.2 & $0.48 \pm 1.34$ & $0.45 \pm 1.29$ & $0.92 \pm 2.83$ & $0.84+2.76$ \\
\hline Yes & 174 & 35.7 & 34 & 19.5 & 7 & 4 & $0.6 \pm 1.57$ & $0.59 \pm 1.54$ & $1.2 \pm 3.56$ & $1.15 \pm 3.53$ \\
\hline
\end{tabular}

\section{Toothbrushing habits}

Once a day

$$
15.2
$$

$0.43 \pm 1.34$

$0.85 \pm 2.96$

$0.78 \pm 2.89$

$0.51 \pm 1.33$

$F=0.47 ; p=0.62$

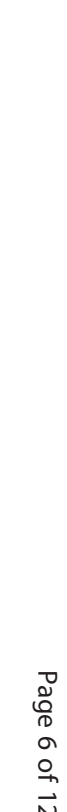


Table 1 ECC pattern according to newborn characteristics, feeding practices, oral hygiene habits, and access to dental services (Continued)

\begin{tabular}{|c|c|c|c|c|c|c|c|c|}
\hline & & & $x^{2}=0.08 ; p=0.772$ & $x^{2}=1.29 ; p=0.256$ & t-test $=-0.93 ; p=0.351$ & t-test $=-1.04 ; p=0.297$ & t-test $=-0.94 ; p=0.35$ & t-test $=-1.08 ; p=0.281$ \\
\hline \multicolumn{9}{|c|}{ Access to dental services } \\
\hline \multicolumn{9}{|c|}{ Dental visit in the previous year } \\
\hline No & 413 & 83.1 & 62 & 1.5 & $0.35 \pm 1.1$ & $0.34 \pm 1.1$ & $0.64 \pm 2.28$ & $0.59 \pm 2.23$ \\
\hline \multirow[t]{2}{*}{ Yes } & 84 & 16.9 & 39.3 & 9.5 & $1.37 \pm 2.24$ & $1.25 \pm 2.17$ & $2.82 \pm 5.2$ & $2.63 \pm 5.17$ \\
\hline & & & $x^{2}=26.6 ; p<0.001$ & $x^{2}=16.61 ; p<0.001$ & t-test $=-6.27 ; p<0.001$ & t-test $=-5.68 ; p<0.001$ & t-test $=-6.13 ; p<0.001$ & t-test $=-5.8 ; p<0.001$ \\
\hline
\end{tabular}


no more than elementary school degree to $18.5 \%$ in those with a university degree $\left(\chi^{2}\right.$ for trend $\left.=5.1 ; \mathrm{p}=0.024\right)$, and for education level of fathers $\left(\chi^{2}\right.$ for trend $=4.52 ; \mathrm{p}=0.03$ ). No significant differences were found according to gender $\left(\chi^{2}=0.87 ; \mathrm{p}=0.352\right)$, father's age $\left(\chi^{2}\right.$ for trend $=0.18 ; \mathrm{p}=$ 0.676 ), and child order ( $\chi^{2}$ for trend $=0.3 ; \mathrm{p}=0.583$ ). Some of the infant feeding practices, such as history and duration of breastfeeding, having experienced sleep with sweetened bottle or pacifier, and time of onset of cup use appear to be related to ECC experience, since ECC prevalence significantly increased from $15.8 \%$ in non breastfed subjects to $30.8 \%$ in children who were breastfed for 20 months or longer $\left(\chi^{2}\right.$ for trend $=4.1 ; \mathrm{p}=0.043$ ), from $17.2 \%$ in those who did not have the habit to go to sleep with sweetened bottle or pacifier to $25.5 \%$ in those who did $\left(\chi^{2}=3.55 ; \mathrm{p}=0.06\right)$, and from $16.2 \%$ to $25 \%$ with increasing age when children started using cups $\left(\chi^{2}\right.$ for trend $=4.44$; $\mathrm{p}=0.035)$. The other feeding/eating practices investigated, that is non-nutritive sucking habits with finger and/or pacifier $\left(\chi^{2}=0.79 ; \mathrm{p}=0.374\right)$, bottle feeding $\left(\chi^{2}=1.47 ; \mathrm{p}=\right.$ 0.225 ) and frequency of sweets consumption (Fisher's exact $\mathrm{p}=0.241$ ) did not show any significant association with ECC. Analogously, none of the oral hygiene habits taken into account, such as frequency $\left(\chi^{2}\right.$ for trend $=0.67$; $\mathrm{p}=0.415)$, time of onset $\left(\chi^{2}\right.$ for trend $\left.=1.57 ; \mathrm{p}=0.21\right)$, and adult supervision of toothbrushing $\left(\chi^{2}=0.02 ; \mathrm{p}=0.899\right)$, as well as toothpaste (Fisher's exact $\mathrm{p}=0.24$ ) and fluoride supplements $\left(\chi^{2}=0.08 ; \mathrm{p}=0.772\right)$ use, seems to influence significantly the risk of ECC in our sample. By contrast, having been visited by a dentist in the previous year is associated with a more than doubled prevalence of ECC, compared to those who did not have access $\left(\chi^{2}=26.6\right.$; $\mathrm{p}<0.001)$.

The associated factors for mean $\mathrm{dmft}$ almost resembled those observed for ECC; however, in some instances, although the trend was similar, statistical significance was different. This was the case for bottle feeding, sleep with a sweetened bottle and/or pacifier, and age at the start of cup use. In particular, mean dmft was significantly lower in children who had experienced bottle feeding ( $\mathrm{t}$-test = 2.66; $\mathrm{p}=0.008$ ), whereas it was not significantly higher in children who used to sleep with sweetened bottle or pacifier ( $\mathrm{t}$-test $=-1.32 ; \mathrm{p}=0.187$ ) and in those who started to use cups later $(\mathrm{F}=1.14 ; \mathrm{p}=0.321)$.

Results on prevalence of S-ECC stratified by several characteristics appear less stable compared to ECC, due to the exiguous number of events. However, a pattern similar to that of ECC was found for most characteristics, although, for the low number of events, statistical significance was not always found. S-ECC increased with increasing age of child, and decreased with increasing age of the mother at the time of the survey and at time of delivery, and decreased with increasing education level of both parents; however, only for mother's age at the time of the survey (Fisher's exact $\mathrm{p}=0.001$ ) and at time of delivery (Fisher's exact $p=0.003$ ), and for mother's education level (Fisher's exact $\mathrm{p}=0.019$ ), the differences were statistically significant at univariate analysis. Among the infant feeding practices, severity of ECC was significantly related to longer duration of breastfeeding (Fisher's exact $\mathrm{p}=$ 0.002), whereas, unexpectedly, S-ECC was significantly lower $(1.9 \%)$ in bottle fed children, compared to those who were not $(7.6 \%)\left(\chi^{2}=7.99 ; \mathrm{p}=0.005\right)$. Among the explored oral hygiene habits, no substantial differences were found according to severity of ECC, except for toothbrushing adult supervision, since S-ECC ranged from $7.7 \%$ in no supervised children to $2.2 \%$ in the supervised ones, although this difference was not statistically significant at the univariate analysis (Fisher's exact $\mathrm{p}=$ 0.128). Finally, S-ECC was unevenly distributed according to visits by a dentist in the previous year, ranging from $1.5 \%$ in those who were not visited to $9.5 \%$ in those who $\operatorname{did}\left(\chi^{2}=16.61 ; \mathrm{p}<0.001\right)$.

Results from the logistic regression models showed that prevalence of ECC significantly increased with age of the child (OR $=1.95 ; 95 \% \mathrm{CI}=1.3-2.91)$, and duration of breastfeeding ( $\mathrm{OR}=1.26 ; 95 \% \mathrm{CI}=1.01-1.57)$, whereas it was significantly lower in children of more educated mothers $(\mathrm{OR}=0.64 ; 95 \% \mathrm{CI}=0.42-0.96)$, and significantly higher in those who had been visited by a dentist in the previous year $(\mathrm{OR}=3.29$; 95\% $\mathrm{CI}=1.72-6.33)$ (Model 1 in Table 2). A similar pattern was found for S-ECC, that significantly increased with breastfeeding $(\mathrm{OR}=2.06$; $95 \%$ $\mathrm{CI}=1.13-3.76$ ), was significantly lower with increasing mothers' education $(\mathrm{OR}=0.16 ; 95 \% \mathrm{CI}=0.05-0.54)$, and was higher in children who had visited a dentist $(\mathrm{OR}=5.54 ; 95 \% \mathrm{CI}=1.15-26.66)$, whereas no significant association was found with children's age (Model 2 in Table 2).

\section{Discussion}

This survey was intended to investigate ECC pattern and severity in a large sample of children in an area in Southern Italy, and to identify factors that may be related to this condition, and that may become the focus for interventions aimed at the prevention of ECC.

Our findings revealed that ECC still represents a consistent burden in our population, interesting almost 20\% of 3-5 years old children, whereas almost $3 \%$ are affected by the more severe disease. In the vast literature aimed at the exploration of ECC prevalence, studies conducted in Europe [40-45] have reported prevalence of ECC ranging from $11.4 \%$ in 3-6 years old Swedish children [46] to $55.1 \%$ in 5 years old children in the Czech Republic [47]. In Italy, reported prevalence of ECC ranged from $8 \%$ [48] to $31.6 \%$ [49], and our figure are similar to many other European and Italian studies [43,48-54]. Only few studies have analysed S-ECC, and a 
Table 2 Results of the logistic regression models

\begin{tabular}{|c|c|c|c|c|}
\hline & OR & SE & $95 \% \mathrm{Cl}$ & $\mathrm{p}$ \\
\hline \multicolumn{5}{|l|}{ Model 1. Outcome: ECC } \\
\hline \multicolumn{5}{|l|}{ Log-likelihood $=-166.48, x^{2}=47.68, p<0.001$} \\
\hline Dental visit in the previous year & 3.29 & 1.1 & $1.72-6.33$ & $<0.001$ \\
\hline Age & 1.95 & 0.4 & $1.3-2.91$ & 0.001 \\
\hline Mother's education level & 0.64 & 0.13 & $0.42-0.96$ & 0.032 \\
\hline History and duration of breastfeeding & 1.26 & 0.14 & $1.01-1.57$ & 0.039 \\
\hline Start using cup & 1.29 & 0.21 & $0.95-1.77$ & 0.103 \\
\hline Sleep with sweetened bottle or pacifier & 1.63 & 0.55 & $0.84-3.16$ & 0.152 \\
\hline Start toothbrushing & 1.16 & 0.25 & $0.76-1.77$ & 0.497 \\
\hline Maternal age at delivery & 0.84 & 0.3 & $0.42-1.71$ & 0.637 \\
\hline Mother's age & 0.99 & 0.36 & $0.49-2.03$ & 0.998 \\
\hline \multicolumn{5}{|l|}{ Model 2. Outcome: S-ECC } \\
\hline \multicolumn{5}{|l|}{ Log-likelihood $=-31.77, x^{2}=22.9, p=0.0063$} \\
\hline Mother's education level & 0.16 & 0.1 & $0.05-0.54$ & 0.003 \\
\hline History and duration of breastfeeding & 2.06 & 0.63 & $1.13-3.76$ & 0.019 \\
\hline Dental visit in the previous year & 5.54 & 4.44 & $1.15-26.66$ & 0.033 \\
\hline Sleep with sweetened bottle or pacifier & 0.61 & 0.7 & $0.06-5.93$ & 0.667 \\
\hline Mother's age & 1.34 & 1.22 & $0.23-7.97$ & 0.748 \\
\hline Start using cup & 1.09 & 0.46 & $0.47-2.51$ & 0.839 \\
\hline Age & 0.91 & 0.46 & $0.34-2.43$ & 0.851 \\
\hline Start toothbrushing & 1.04 & 0.56 & $0.36-2.98$ & 0.938 \\
\hline Maternal age at delivery & 0.3 & 0.27 & $0.05-1.81$ & 0.188 \\
\hline
\end{tabular}

$\overline{E C C}=$ early childhood caries, S-ECC $=$ severe early childhood caries.

prevalence of $6.5 \%$ has been reported in 3 years old children in Lithuania [55], and of $12.2 \%$ in $2-3$ years old children in Sweden [41]. It should be pointed out, however, that these studies have been conducted with differing age ranges, methods, definitions of ECC, and time frame, and therefore, comparisons must be interpreted cautiously.

As in most studies aimed at the investigation of the oral health of pre-school children, we found a high proportion of caries-free subjects and a mean dmft of 0.5 , whereas the pattern and severity of caries are polarized to certain subgroups of the population resembling their socioeconomic status [7,40,52]. Moreover, in children with caries the $\mathrm{dmft}$ tends to be high, reaching a mean of 6.86 in those with S-ECC. Indeed, when we analyzed the role of several conditions that are known or controversial factors related to ECC pattern and severity, we found that ECC was not evenly distributed in the population, but it is substantially influenced by social demographics and infant feeding practices: although the overall picture on prevalence of ECC and S-ECC does not appear particularly concerning, a more detailed description of this phenomenon through stratification according to several characteristics, shows significant differences across subgroups of the population. This circumstance is alarming, considering that most differences, as reported in previous studies, are related to social demographics $[8,12,15,20]$ and infant feeding practices $[18,19]$, and therefore, place for improvement is substantial. The finding that the overall ECC prevalence of about $20 \%$, in line with with the $80 \%$ caries free at age 6 target set by WHO within the Health21 policy framework [56], becomes about 30\% in older (5 years) children, in children born from younger mothers at the time of survey and of delivery, and who had a lower education level poses attention on the role that social and cultural factors may have on the occurrence of ECC, delineating dramatic differences across social groups. Analogously, the difference of about $6-10$ points compared to the overall ECC prevalence according to some feeding practices, underscores the need for more effective interventions to promote prevention in the field of oral health in infancy. In particular, since the significantly higher prevalence was found in children who were longer breastfed, who used to go to sleep with sweetened bottle or pacifier and later began to drink from cups, it appears that a strategic role in the prevention of ECC may be played by pediatricians, since they are the first health 
care professionals who are contacted by mothers, and represent the reference figure for counseling on feeding practices. This acquires even more substance in the Italian context, since every newborn is assigned a pediatrician free of charge within the NHS, that represents the "gate keeper" for all other accesses to health care within the NHS. However, a recent study conducted by one of us on knowledge, attitudes, and practices of pediatricians regarding prevention of oral diseases in Italy raised the issue of lack of knowledge of the main associated factors for oral diseases, although almost all pediatricians believed that they had an important responsibility in preventing oral diseases in children and provided an oral examination on their patients [57]. Moreover, the statistical association between breastfeeding duration and ECC must be interpreted cautiously since it is important to consider that the present study included children who were no longer breastfed at the time of examination. This may have biased results as caries presence 3-4 years after breastfeeding has ceased might not be attributed to breastfeeding. Moreover, it should be considered that exclusive breastfeeding is only performed during the first 6 months of life, and subsequent introduction of new foods, especially those rich in sucrose, is a confounding factor when analyzing the association between breastfeeding and caries [31].

Moreover, the finding that children with ECC and S-ECC are significantly more likely to have visited a dentist in the previous year suggests that dentists are contacted only after ECC has occurred; therefore, it seems that dentists do not play an effective role in the prevention practices.

Unexpectedly, we did not find an association between ECC or S-ECC and bottle feeding, whereas it is well-known that particularly severe disease has long been called "nursing caries" or "bottle caries". However, we may hypothesize that it is not bottle feeding per se that represents an associated factor for ECC and S-ECC, but, more consistently, the consumption, through nursing bottles, of sweetened liquids, and indeed, our study clearly showed the importance of sweetened bottle or pacifier, particularly at night, as possible associated factors for ECC and S-ECC.

It is well-known that oral hygiene practices are among the most effective measures in the prevention of dental caries, and we found that only toothbrushing adult supervision was associated with a significantly lower risk of S-ECC. This is not surprising, since in young children oral hygiene is almost completely a parents' responsibility, whereas simple assessment of frequency of tooth brushing may not be a valid indicator of oral hygiene.

\section{Limitations}

We used a self-administered questionnaire to register all potential associated factors. This could have determined a shift to more socially desirable answers, even though this does not appear to be the case in our study, since even self-reporting demonstrated a wide-spread diffusion of associated factors in our population. Moreover, the cross-sectional nature of the study does not allow any cause-effect relationship, since data on "associated factors" and "outcomes" are assessed at the same time. Nonetheless, this study represents a useful way to determine the prevalence of ECC and S-ECC and, eventually, to identify differences among subgroups disaggregated by social demographics, infant feeding practices, oral hygiene habits, and access to dental services, in order to target preventive interventions on those subjects that manifest poorer oral health. As with any survey based on a selfadministered questionnaire, information resulting from the memory of parents may not be entirely accurate, primarily because of the long time frame used in the study that may have introduced recall bias. On the other hand, longer time frames are useful for formulating broad prevalence estimates in a context in which no data are available. Finally, problems regarding representativeness and generalizability should be taken into account, and we believe that the sampling methods and the high response rate allows us to be confident on the representativeness of the chosen sample and that our results may be generalized to children living in the Southern part of Italy.

\section{Conclusions}

In conclusion, results of our study demonstrate that even in western countries ECC and S-ECC still represent a significant burden in preschool children, particularly in those disadvantaged, and that most of the known modifiable associated factors regarding feeding practices and oral hygiene are still very spread in the population. Paediatricians and dentists should play a more effective role in the prevention of ECC.

\section{Competing interests}

The authors declare that they have no competing interests.

\section{Authors' contributions}

CGAN and MP designed and coordinated the study. LF performed dental examinations. $A B, L F$ and $C P$ contributed to the data analysis and results interpretation. MP wrote the manuscript. All authors read and approved the final manuscript.

Received: 7 January 2014 Accepted: 19 February 2014

Published: 27 February 2014

\section{References}

1. World Health Organization: The World Oral Health Report 2003: Continuous Improvement of Oral Health in the 21st Century - the Approach of the WHO Global Oral Health Programme. Geneva: World Health Organization; 2003.

2. Martins-Júnior PA, Vieira-Andrade RG, Corrêa-Faria P, Oliveira-Ferreira F, Marques LS, Ramos-Jorge ML: Impact of early childhood caries on the oral health-related quality of life of preschool children and their parents. Caries Res 2013, 47:211-218.

3. Hallett KB, O'Rourke PK: Social and behavioural determinants of early childhood caries. Aust Dent J 2003, 48:27-33.

4. American Academy on Pediatric Dentistry, American Academy of Pediatrics: Policy on Early Childhood Caries (ECC): Classifications, Consequences, and Preventive Strategies. Pediatr Dent 2008-2009, 30:40-43. 
5. Harris $R$, Nicoll $A D$, Adiari PM, Pine CM: Risk factors for dental caries in young children: a systematic review of the literature. Community Dent Health 2004, 21:71-85.

6. Skeie MS, Riordan PJ, Klock KS, Espelid I: Parental risk attitudes and caries related behaviours among immigrant and western native children in Oslo. Community Dent Oral Epidemiol 2006, 34:103-113.

7. Bonanato K, Paiva SM, Pordeus IA, Ramos-Jorge ML, Barbabela D, Allison PJ: Relationship between mothers' sense of coherence and oral health status of preschool children. Caries Res 2009, 43:103-109.

8. Wigen TI, Espelid I, Skaare AB, Wang NJ: Family characteristics and caries experience in preschool children. A longitudinal study from pregnancy to 5 years of age. Community Dent Oral Epidemiol 2011, 39:311-317

9. Dye BA, Vargas CM, Lee JJ, Magder L, Tinanoff N: Assessing the relationship between children's oral health status and that of their mothers. J Am Dent Assoc 2011, 142:173-183.

10. Wigen Tl, Wang NJ: Caries and background factors in Norwegian and immigrant 5-year-old children. Community Dent Oral Epidemiol 2010, 38:19-28.

11. Laaksonen M, Rahkonen O, Karvonen S, Lahelma E: Socioeconomic status and smoking: analysing inequalities with multiple indicators. Eur J Public Health 2005, 15:262-269.

12. Aida J, Ando Y, Oosaka M, Niimi K, Morita M: Contributions of social context to inequality in dental caries: a multilevel analysis of Japanese 3-year-old children. Community Dent Oral Epidemiol 2008, 36:149-156.

13. Meurman PK, Pieniha"kkinen K: Factors associated with caries increment: a longitudinal study from 18 months to 5 years of age. Caries Res 2010, 44:519-524.

14. Gao XL, Hsu CYS, Xu YC, Loh T, Koh D, Hwarng HB: Behavioral pathways explaining oral health disparity in children. J Dent Res 2010, 89:985-990.

15. Piovesan C, Mendes FM, Ferreira FV, Guedes RS, Ardenghi TM: Socioeconomic inequalities in the distribution of dental caries in Brazilian preschool children. J Public Health Dent 2010, 70:319-326.

16. Nunn ME, Dietrich T, Singh HK, Henshaw MM, Kressin NR: Prevalence of early childhood caries among very young urban Boston children compared with US children. J Public Health Dent 2009, 69:156-162.

17. Mennella JA, Beauchamp GK: Flavor experiences during formula feeding are related to preferences during childhood. Early Hum Dev 2002, 68:71-82.

18. Kawashita Y, Kitamura M, Saito T: Early childhood caries. Int J Dent 2011. http://dx.doi.org/10.1155/2011/725320.

19. Menghini $G$, Steiner M, Thomet E, Roos M, Imfeld T: Caries prevalence in 2-year-old children in the city of Zurich. Community Dent Health 2008, 25:154-160

20. Tyagi R: The prevalence of nursing caries in Davangere preschool children and its relationship with feeding practices and socioeconomic status of the family. J Indian Soc Pedod Prev Dent 2008, 26:153-157.

21. Du M, Luo Y, Zeng X, Alkhatib N, Bedi R: Caries in preschool children and its risk factors in 2 provinces in China. Quintessence Int 2007, 38:143-151.

22. Corrêa-Faria P, Martins-Júnior PA, Vieira-Andrade RG, Marques LS, RamosJorge ML: Factors associated with the development of early childhood caries among Brazilian preschoolers. Braz Oral Res 2013, 27:356-362.

23. Al-Jewair TS, Leake JL: The prevalence and risks of early childhood caries (ECC) in Toronto, Canada. J Contemp Dent Pract 2010, 11:1-8.

24. Cogulu D, Ersin NK, Uzel A, Eronat N, Aksit S: A long-term effect of caries-related factors in initially caries-free children. Int J Paediatr Dent 2008, 18:361-367.

25. Nunes AMM, Alves CMC, Araùjo FB, Ortiz TML, Ribeiro MRC, Silva AAM, Ribeiro CCC: Association between prolonged breast-feeding and early childhood caries: a hierarchical approach. Community Dent Oral Epidemiol 2012, 40:542-549.

26. Declerck D, Leroy R, Martens L, Lesaffre E, Garcia-Zattera MJ, Vanden Broucke S, Debyser M, Hoppenbrouwers K: Factors associated with prevalence and severity of caries experience in preschool children. Community Dent Oral Epidemiol 2008, 36:168-178.

27. Seow WK, Clifford H, Battistutta D, Morawska A, Holcombe T: Case-control study of early childhood caries in Australia. Caries Res 2009, 43:25-35.

28. Begzati A, Berisha M, Meqa K: Early childhood caries in preschool children of Kosovo-a serious public health problem. BMC Public Health 2010, 10:788-895.

29. Hallett KB, O'Rourke PK: Pattern and severity of early childhood caries. Community Dent Oral Epidemiol 2006, 34:25-35.
30. Tiberia MJ, Milnes AR, Feigal RJ, Morley KR, Richardson DS, Croft WG, Cheung WS: Risk factors for early childhood caries in Canadian preschool children seeking care. Pediatr Dent 2007, 29:201-208.

31. Van Palenstein Helderman WH, Soe W, Van't Hof MA: Risk factors of early childhood caries in a Southeast Asian population. J Dent Res 2006, 85:85-88.

32. Leroy R, Hoppenbrouwers K, Jara A, Declerck D: Parental smoking behaviour and caries experience in preschool children. Community Dent Oral Epidemiol 2008, 36:249-257.

33. Tsai Al, Chen CY, Li LA, Hsiang CL, Hsu KH: Risk indicators for early childhood caries in Taiwan. Community Dent Oral Epidemiol 2006, 34:437-445.

34. Angelillo IF, Anfosso R, Nobile CGA, Pavia M: Prevalence of dental caries in schoolchildren in Italy. Eur J Epidemiol 1998, 14:351-357. Erratum 1998, 14:733.

35. Angelillo IF, Torre I, Nobile CGA, Villari P: Caries and fluorosis prevalence in communities with different concentrations of fluoride in the water. Caries Res 1999, 33:114-122.

36. Nobile CGA, Pavia M, Fortunato L, Angelillo IF: Prevalence and factors related to malocclusion and orthodontic treatment need in children and adolescents in Italy. Eur J Public Health 2007, 17:637-641.

37. Bianco A, Fortunato L, Nobile CGA, Pavia M: Prevalence and determinants of oral impacts on daily performance: results from a survey among school children in Italy. Eur J Public Health 2010, 20:595-600.

38. World Health Organization: Oral Health Surveys: Basic Methods. 4th edition. Geneva: World Health Organization; 1997.

39. Stata Corporation: Stata Statistical Software. Release 11.1 College Station, Texas 77845 USA, 2009.

40. Robke FJ: Effects of nursing bottle misuse on oral health. Prevalence of caries, tooth malalignments and malocclusions in North-German preschool children. J Orofac Orthop 2008, 69:5-19.

41. Bankel M, Eriksson UC, Robertson A, Köhler B: Caries and associated factors in a group of Swedish children 2-3 years of age. Swed Dent J 2006, 30:137-146.

42. Droz D, Guéguen R, Bruncher P, Gerhard JL, Roland E: Epidemiological study of oral dental health of 4-year-old children in French nursery schools. Arch Pediatr 2006, 13:1222-1229.

43. Ferro R, Besostri A, Meneghetti B, Beghetto M: Comparison of data on Early Childhood Caries (ECC) with previous data for Baby Bottle Tooth Decay (BBTD) in an Italian kindergarten population. Eur J Paediatr Dent 2004, 5:71-75.

44. Wennhall I, Matsson L, Schröder U, Twetman S: Caries prevalence in 3-year-old children living in a low socio-economic multicultural urban area in southern Sweden. Swed Dent J 2002, 26:167-172.

45. Dimitrova MM, Kukleva MP, Kondeva VK: Prevalence of early childhood caries and risk factors in children from 1 to 3 years of age in Plovdiv, Bulgaria. Folia Med 2002, 44:60-63.

46. Stromberg U, Holmen A, Magnusson K, Twetman S: Geo-mapping of time trends in childhood caries risk - a method for assessement of preventive care. BMC Oral Health 2012, 12:9-15.

47. Lencova E, Pikhart $H$, Broukal Z: Early childhood caries trend surveillance shortcomings in the Czech Republic. BMC Public Health 2012, 12:547.

48. Panetta F, Dall'Oca S, Nofroni I, Quaranta A, Polimeni A, Ottolenghi L: Early childhood caries. Oral health survey in kindergartens of the 19th district in Rome. Minerva Stomatol 2004, 53:669-678.

49. Ferro R, Besostri A, Olivieri A, Stellini E, Mazzoleni S: Preschoolers' dental caries experience and its trend over 20 years in a North-East Italian health district. Eur J Paediatr Dent 2007, 8:199-204.

50. Ferro R, Besostri A, Meneghetti B, Stellini E: Prevalence and severity of dental caries in 5- and 12-year old children in the Veneto Region (Italy). Community Dent Health 2007, 24:88-92.

51. Campus G, Lumbau A, Sanna AM, Solinas G, Lugliè P, Castiglia P: Oral health condition in an Italian preschool population. Eur J Paediatr Dent 2004, 5:86-91.

52. Ferro R, Cecchin C, Besostri A, Olivieri A, Stellini E, Mazzoleni S: Social differences in tooth decay occurrence in a sample of children aged 3 to 5 in north-east Italy. Community Dent Health 2010, 27:163-166.

53. Campus G, Solinas G, Strohmenger L, Cagetti MG, Senna A, Minelli L, Majori S, Montagna MT, Reali D, Castiglia P: National pathfinder survey on children's oral health in Italy: pattern and severity of caries disease in 4-year-olds. Caries Res 2009, 43:155-162.

54. Petti S, Cairella G, Tarsitani G: Rampant early childhood dental decay: an example from Italy. J Public Health Dent 2000, 60:159-166. 
55. Slabsinskiene E, Milciuviene S, Narbutaitè J, Vasiliauskienè I, Andruskeviciené $V$, Bendoraitienè EA, Saldunaitè K: Severe early childhood caries and behavioral risk factors among 3-year-old children in Lituania. Medicina 2010, 46:135-141.

56. HEALTH 21: An introduction to the health for all policy framework fort the WHO European Region. Copenhagen: WHO regional Office for Europe; 1998.

57. Di Giuseppe G, Nobile CGA, Marinelli A, Angelillo IF: Knowledge, attitude and practices of pediatricians regarding the prevention of oral diseases in Italy. BMC Public Health 2006, 6:176-184.

doi:10.1186/1471-2458-14-206

Cite this article as: Nobile et al.: Pattern and severity of early childhood caries in Southern Italy: a preschool-based cross-sectional study. BMC Public Health 2014 14:206.

\section{Submit your next manuscript to BioMed Central and take full advantage of:}

- Convenient online submission

- Thorough peer review

- No space constraints or color figure charges

- Immediate publication on acceptance

- Inclusion in PubMed, CAS, Scopus and Google Scholar

- Research which is freely available for redistribution 